\title{
STEREOSCOPIC OBSERVATION OF SYNTHETIC DIAMOND WITH HARUTA STEREO-PAIRS OF SYNCHROTRON TRANSMISSION DOUBLE-CRYSTAL TOPOGRAPHS
}

\author{
M. MOORE ${ }^{a}$, A.R. LANG ${ }^{b}$ AND W. WIERZCIIOWSKI ${ }^{a *}$ \\ a Department of Physic, Royal IIolloway College, University of London \\ Egham IIill, Egham, Surrey, TW20 0EX, Great Britain \\ ${ }^{6}$ University of Bristol, II.H. Wills Physics Laboratory \\ Royal Fort, Tyndall Ave., Bristol BS8 1TL, Great Britain
}

The IIaruta method is used for stereoscopic observation of a $1.5 \times 4 \times 4 \mathrm{~mm}^{3}$ cuboctahedral synthetic diamond with transmission double-crystal topography. The experiments were performed using a synchrotron double-crystal arrangement with asymmetrical diamond 220 reflection selecting $1.0 \AA$ radiation. The appropriate IIaruta pairs were matched from a series taken with positions on the rocking curve changed by small intervals. A reasonable stereoscopic effect was obscrved for most defect images, particularly dislocations, stacking faults and growth sector boundaries. It was established that some interference fringes do not produce good stereoscopic effects and appear on the exit surfaces of the diamond.

PACS numbers: 61.10.-i

\section{Introduction}

The stereoscopic techniques offer a great help in resolving of the configuration of defects. A very convenient method of producing the stereoscopic effect in X-ray topographis was proposed by IIaruta [1]. This method is usually applied for transmission single-crystal topographs and it consists of taking a pair of topographs differing by a small rotation about the difraction vector, symmetrically around the central position. The suitable angle of rotation is dependent on the actual thickness of the crystal [2]. The advantage of the method is the use of topographs with equivalent contrast.

In the present work the II aruta-pair technique is used for transmission synchrotron double-crystal topography of diamond. With low absorption, the transmission double-crystal topographs contain a significant contribution of direct diffraction contrast. In comparison with transmission single-crystal topographs, they differ by their much greater spatial extension of defect images and in considerable

*Present and permanent address: Institute of Electronic Materials Technology, Wólczyniska 133, 01-919 Warszawa, Poland. 
dependence of the image on position on the rocking curve. The double-crystal topographs also exhibit numerous interference fringes, whose visibility increases with reduction of the incident divergence.

The purpose of the present experiment is the study of stereoscopic effects in transmission double-crystal topography and localization of particular elements in the topographic image. A particular interest is also observation of the stereoscopic effects in the interference fringes. The results of the double-crystal investigation were compared with synchrotron single-crystal IIaruta pairs, and with sections topographs.

\section{Experimental}

The investigated sample was a $1.5 \times 4 \times 4 \mathrm{~mm}^{4}$ slab prepared from a synthetic diamond grown by Sumitomo Electric Company, by polishing out of the upper and down parts. The sample was kindly loaned to us by Dr. A.T. Collins. The crystal contained two artificially introduced surfaces perpendicular to the main [001] growth direction. The investigation of the present diamond with other techniques is described in Ref. [3].

The actual topographic investigation was performed at the station 7.6 of the S.R.S. synchrotron source at Daresbury Laboratory. The double-crystal topographs were exposed using an arrangement with the asymmetrical 220 reflection at the investigated diamond, matched by the 331 reflection on a (110) oriented silicon monochromator. The arrangement selected $1.0 \AA$ radiation.

In the present experiment the tilting angle of rotation about the diffraction vector was equal to $\pm 2.5^{\circ}$. For each of the two tilt positions series of topographs were taken with angular setting altered in steps of $0.5^{\prime \prime}$, and more than 18 topographs were exposed in one passage through the peak. The small angular step minimized the changes of the images and we were able to match pairs corresponding to equivalent points on the rocking curve, providing a good stereoscopic effect.

For comparison we also took the synchrotron single-crystal IIaruta-pair of topographs in equivalent geometry and with the same $1.0 \AA$ radiation.

\section{Experimental results and discussion}

A representative Haruta-pair of double-crystal topographs of the Sumitomo diamond, which may be used for stereoscopic observation, is shown in Fig. 1. These topographs can be used for stereoscopic observation using a single lens with 3-4 cm focal length in front of each eye. An equivalent IIaruta-pair of single crystal projection topographs is shown in Fig. 2. For better understanding of the topographs a diagram presenting the corresponding projection of the diamond and the position of the diffraction vector is shown in Fig. 3. The diffracted beam exits mainly through the artificial (001) face further from the beam, and the topographs revealed dominantly the defects closer to this side of the crystal.

The defect structure of the sample was discussed in detail in our previous paper [3]. In the present topographs we observe approximately half of the dislocations present in the crystal, grouped preferentially in some bundles. The dislocations bundles are related to some growth sectors according to the rule that the preferred orientation of a grown-in dislocation is perpendicular to the growing face. The dislocations seem to emerge from the seed, which was situated approximately $1 \mathrm{~mm}$ from the actual face of the crystal. The present diamond contains a number of stacking faults, grouped mainly close to octahedral faces. 

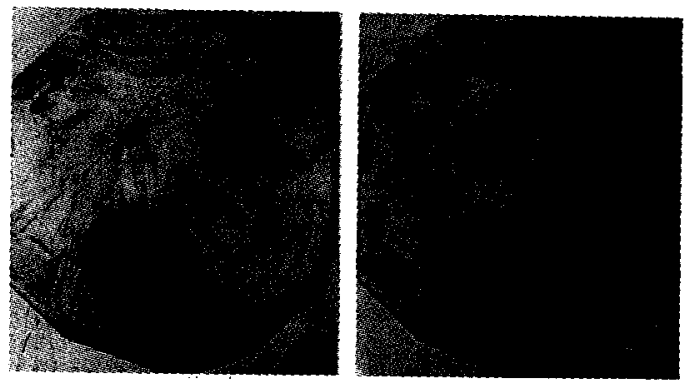

Fig. 1
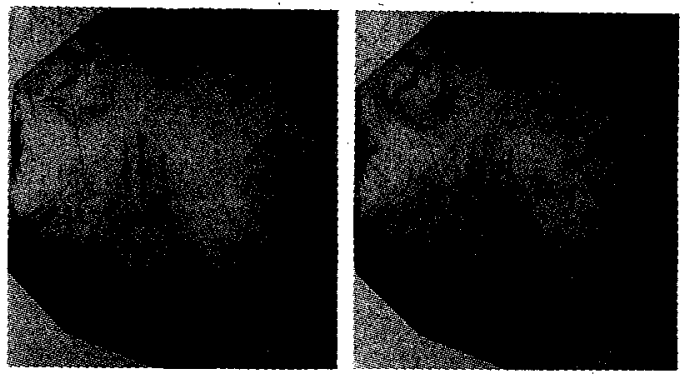

Fig. 2

Fig. 1. A representative IIaruta stercoscopic pair of synchrotron double-crystal transmission topographs of Sumitomo synthetic diamond, taken in the asymmetric 220 reflection of $1.0 \AA$ radiation. The stereoscopic effect may be achicved using a stereoscopic viewer with lenses of focal length $3-4 \mathrm{~cm}$.

Fig. 2. IIaruta-pair of synchrotron single-crystal topographs of the Sumitomo diamond taken in the same 220 reflection of $1.09 \AA$ radiation.

It may be noticed that the images of the defects in the double-crystal topographs are much more intense than in the single-crystal topographs, and contain more interference fringes. In particular the fringes form dotting along the dislocation lines that is invisible in the single-crystal topographs. The fringes on the stacking faults are visible in both methods, but those in the double-crystal topographs are more distinct, and are dependent on angular setting.

The diamond contains a complex pattern of growth sectors, with a number of sectors surrounding a major central (001) sector. The single-crystal topographs revealed only a part of the growth-sector boundary outcrops on the artificially polished surfaces. These boundaries are marked with thin lines in Fig. 3. In addition, the double-crystal topographs revealed interference fringes corresponding to the boundaries of the central growth-sector inside the crystal. The above-mentioned defects produce a good stereoscopic effect. That applies also to the fringes along dislocations and to the fringes on the stacking faults, and the boundaries of the central sector.

Our particular attention was devoted to a system of fringes that appeared in the upper right part of the fringes shown in Fig. 1. In stereoscopic observation this systems appeared as localized on the exit surface. The analysis of all topographic investigations performed points that the system of fringes is due to a boundary between $\{100\}$ and $\{011\}$ growth sectors, and a stacking fault. These objects are situated near to the other surface, closer to the seed. 

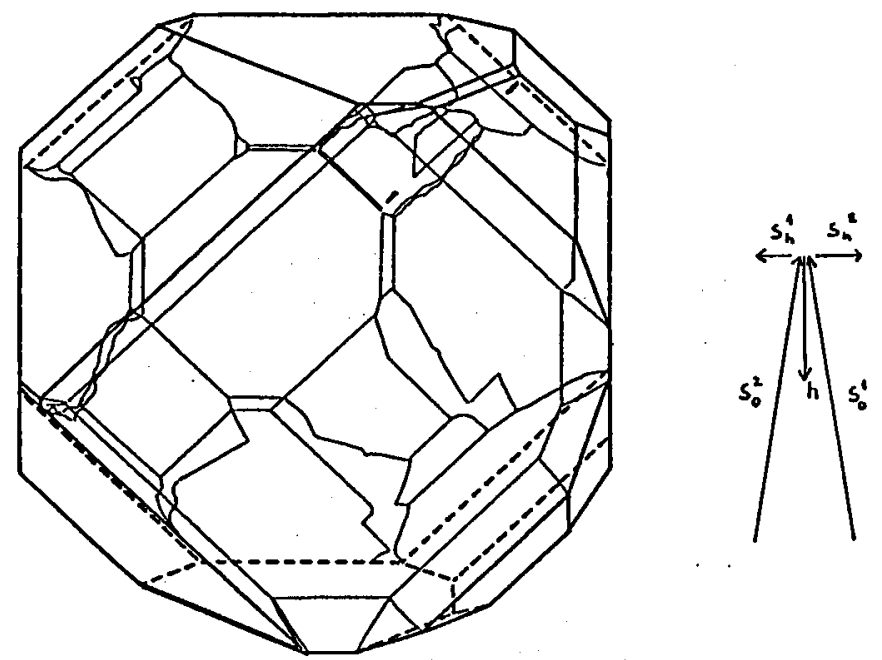

Fig. 3. Drawing of Sumitomo diamond in perspective corresponding to that of the topographs in Figs. 1 and 2, with the direction of the incident and reflected beam shown. For identification of details, the growth sector boundaries on the polished surfaces are marked by thin lines.

The appearance of the fringes on the exit surface is explainable noticing that a good stereoscopic effect is obtained when the details of image are reproduced by radiation propagating inside the crystal close to the direction of the reflected beam. The interference fringes can be formed by radiation propagating inside the crystal along directions far from that of the reflected beam and not producing a good stereoscopic effect. That can take place especially for the fringes formed due to the interference of the wave-fields with excitation points situated close to the centre of dispersion hyperbola.

\section{Conclusions}

A IIaruta-pair technique is applied for stereoscopic observation of diamond in double-crystal topographs. Suitable pairs, corresponding to equivalent points on the rocking curve, were matched from series of topographs taken with angular setting altered by small steps.

The attaimment of a good stereoscopic effect of most defects is of high practical importance in view of the high sensitivity and the revelation of more details than in the case of single-crystal topography.

An example of appearance if interference fringes, far from their origin, pointing the propagation of wave-fields far from the reflected beam direction, was found.

\section{References}

[1] K. IIaruta, J. Appl. Phys. 36, 1789 (1965).

[2] A.R. Lang, in: Modern Diffraction and Imaging Techniques, Eds. S. Amelinckx, R. Gevers, G. Rimaut, J. Van Landuyt, North-IIolland, Amsterdam 1970, p. 407.

[3] W.K. Wierzchowski, M. Moore, A. Makepeace, Yacoot, J. Cryst. Growth 114, 209 (1991). 